Correction

\title{
Correction to: Much Ado About Nothing? An Analysis of Prioritization at Six Canadian Universities
}

Donna Kotsopoulos ${ }^{\mathrm{a}}$ (1), Joanne McKee ${ }^{\mathrm{b}}$, Vianne Timmons ${ }^{\mathrm{c}}$, Victoria Gisondi ${ }^{\mathrm{e}}$, Tina Goebel ${ }^{\mathrm{e}}$, Brandon Verkerk ${ }^{\mathrm{d}}$, Stephen King ${ }^{\mathrm{c}}$, Lisa Keeping ${ }^{\mathrm{f}}$, Mary Kelly ${ }^{\mathrm{f}}$ and Ruth Cruikshank ${ }^{\mathrm{f}}$

${ }^{a}$ Faculty of Arts and Social Science, Huron University College, London, Canada.

E-mail: dkotsopo@gmail.com

${ }^{\mathrm{b}}$ Ryerson University, Toronto, Canada.

E-mail: joanne.mckee@ryerson.ca

${ }^{c}$ University of Regina, Regina, Canada.

E-mail: Vianne.timmons@uregina.ca, Stephen.king@uregina.ca

${ }^{\mathrm{d}}$ Faculty of Science, Wilfrid Laurier University, Waterloo, Canada.

E-mail: bverkerk@gmail.com

${ }^{\mathrm{e}}$ Faculty of Education, Wilfrid Laurier University, Waterloo, Canada.

E-mail: victoriagisondi@hotmail.com,tgoebel@uguelph.ca

${ }^{\mathrm{f}}$ Lazaridis School of Business and Economics, Wilfrid Laurier University, Waterloo, Canada.

E-mail: lkeeping@wlu.ca, mkelly@wlu.ca, rcruikshank@wlu.ca

Higher Education Policy (2021) 34, 540-541. https://doi.org/10.1057/s41307-01900146-0; published online 3 June 2019

\section{Correction to: Higher Education Policy (2019) https://doi.org/10.1057/s41307-018-00132-y}

Unfortunately, due to overlooking Table 3 was inserted twice. Below please find the missing Table 4. 
Donna Kotsopoulos et al.

Much Ado About Nothing?

Table 4 Financial metrics at the University of Saskatchewan throughout 2011-2015

\begin{tabular}{llllll}
\hline & 2011 & 2012 & 2013 & 2014 & 2015 \\
\hline Net Income/Loss (\%) & 18.5 & 4.9 & 10.8 & 14.7 & 12.0 \\
Net Operating Revenues (\%) & 16.2 & 13.8 & 9.6 & 11.9 & 11.0 \\
Primary Reserve (\%) & 37.4 & 35.0 & 39.0 & 44.0 & 48.7 \\
Interest Burden (\%) & 0.9 & 2.9 & 0.6 & 0.8 & 0.8 \\
Viability (\%) & $14,480.2$ & $16,360.7$ & 546.6 & 715.6 & 286.6 \\
\hline
\end{tabular}

